\title{
The multi-valued domination of metric spaces
}

\author{
Mirosław Ślosarski
}

Correspondence: slosmiro@gmail. com

Technical University of Koszalin, Śniadeckich 2, Koszalin PL-75-453, Poland

\author{
Abstract \\ In this article, we introduce the notion of multi-valued domination of metric spaces, \\ which is a generalization of domination in the sense of Borsuk and which \\ encompasses previous generalizations. \\ AMS classification: 32A12; 47H10; 55M20; 54C55; 54C15.
}

Keywords: multi-domination, absolute neighborhood multi-retracts, admissible multiretract, Lefschetz space, movable space

\section{Introduction}

In 1967, Borsuk (see, [1]) introduced the notion of the domination of space in the class of metric spaces, i.e., a metric space $Y$ dominates a metric space $X$ (we write $Y \geq \mathrm{X}$ ) if there are continuous maps $f: Y \rightarrow X, g: X \rightarrow Y$ such that for every $x \in X f(g(x))=x$. He has proven that the domination of space retains the fixed point property, i.e., if $Y$ dominates the space of $X$ and if $Y$ is a Lefschetz space, then $X$ is also a Lefschetz space. In 1983, Suszycki (see, [2]) introduced the notion of multi-retract and as a consequence he defined absolute multi-retracts $(m-A R)$ and absolute neighborhood multi-retracts $(m-A N R)$. On the basis of these notions (multi-retracts) we can consider a certain domination of space, i.e., a space $Y$ dominates an $X$ space (in the sense of Suszycki) if there exist a multi-valued u.s.c. map $\phi: Y \rightarrow X$ with compact images (for every $y \in Y \phi(y)$ has a trivial shape, (see, [2,3])) and a continuous map $g: X \rightarrow Y$ such that for every $x \in X x \in \phi(g(x))$ (see, [2]). An easy example (see, 3.7) shows that the domination of space in the sense of Suszycki does not retain the fixed point property. It is not difficult to notice that the domination in the sense of Suszycki is the generalization of the domination in the sense of Borsuk. In 2008 we introduced the notions (see, [4]) of absolute multi-retracts $(A M R)$ and the absolute neighborhood multi-retracts $(A N M R)$. On the basis of these notions we can also consider a certain kind of domination of space, i.e., a space $Y$ dominates a space $\mathrm{X}$ in a multi-valued sense (see, [4]) if there exists a continuous mapping $f: Y \rightarrow X$ and a multi-valued admissible mapping $\phi: X \rightarrow Y$ such that for every $x \in X f(\phi(x))=\{x\}$. We will show that it is the generalization of Borsuk domination that retains the fixed point property.

\section{Preliminaries}

Throughout this article all topological spaces are assumed to be metric. Let $H_{*}$ be the Čech homology functor with compact carriers and coefficients in the field of rational numbers $\mathbb{Q}$ from the category of Hausdorff topological spaces and continuous maps to the category of graded vector spaces and linear maps of degree zero. Thus $H_{*}(X)=\left\{H_{q}\right.$

(C) 2012 Ślosarski; licensee Springer. This is an Open Access article distributed under the terms of the Creative Commons Attribution License (http://creativecommons.org/licenses/by/2.0), which permits unrestricted use, distribution, and reproduction in any medium, provided the original work is properly cited. 
$(X)\}$ is a graded vector space, $H_{q}(X)$ being the $q$-dimensional Cech homology group with compact carriers of $X$. For a continuous map $f: X \rightarrow Y, H_{*}(f)$ is the induced linear $\operatorname{map} f_{*}=\left\{f_{q}\right\}$, where $f_{q}: H_{q}(X) \rightarrow H_{q}(Y)$ [5]. A space $X$ is acyclic if:

(i) $X$ is non-empty,

(ii) $H_{q}(X)=0$ for every $q \geq 1$ and

(iii) $H_{0}(X) \approx \mathbb{Q}$.

A continuous mapping $f: X \rightarrow Y$ is called proper if for every compact set $K \subset Y$ the set $f^{1}(K)$ is non-empty and compact. A proper map $p: X \rightarrow Y$ is called Vietoris provided for every $y \in \mathrm{Y}$ the set $p^{-1}(y)$ is acyclic. Let $X$ and $Y$ be two spaces and assume that for every $x \in X$ a non-empty subset $\phi(x)$ of $Y$ is given. In such a case we say that $\phi: X \multimap Y$ is a multi-valued mapping. For a multi-valued mapping $\phi: X \multimap Y$ and a subset $U \subset Y$, we let:

$$
\varphi^{-1}(U)=\{x \in X ; \varphi(x) \subset U\} .
$$

If for every open $U \subset Y$ the set $\phi^{-1}(U)$ is open, then $\phi$ is called an upper semi-continuous mapping; we shall write that $\phi$ is u.s.c.

Let $\phi: X \rightarrow Y$ be a multi-valued map. A pair $(p, q)$ of single-valued, continuous maps is called a selected pair of $\phi$ (written $(p, q) \subset \phi$ ) if there exists a metric space $Z$ such that the following two conditions are satisfied:

(i) $p: Z \rightarrow X$ is a Vietoris map,

(ii) $q\left(p^{-1}(x)\right) \subset \phi(x)$ for any $x \in \mathrm{X}$, where $q: Z \rightarrow Y$ is a continuous map.

Definition 2.1. A multi-valued mapping $\phi: X \rightarrow Y$ is called admissible provided there exists a selected pair $(p, q)$ of $\phi$.

Proposition 2.2. (see, [5]) If $\phi: X \multimap Y$ and $\psi: Y \rightarrow T$ are admissible, then the composition $\psi \circ \phi: X \rightarrow T$ is admissible and for every $\left(p_{1}, q_{1}\right) \subset \phi$ and $\left(p_{2}, q_{2}\right) \subset \psi$ there exists a pair $(p, q) \subset \psi \circ \phi$ such that $q_{2_{*}} p_{2_{*}}^{-1} \circ q_{1_{*}} p_{1_{*}}^{-1}=q_{*} p_{*}^{-1}$.

Let $\phi: X \rightarrow X$ be an admissible map. Let $(p, q) \subset \phi$, where $p: Z \rightarrow X$ is a Vietoris mapping and $q: Z \rightarrow X$ a continuous map. Assume that $q_{*} \circ p_{*}^{-1}: H_{*}(X) \rightarrow H_{*}(X)$ is a Leray endomorphism for all pairs $(p, q) \subset \phi$. For such a $\phi$, we define the Lefschetz set $\Lambda(\phi)$ of $\phi$ by putting $\Lambda(\varphi)=\left\{\Lambda\left(q_{*} p_{*}^{-1}\right) ;(p, q) \subset \varphi\right\}$.

Definition 2.3. An admissible map $\phi: X \rightarrow X$ is called a Lefschetz map provided the Lefschetz set $\Lambda(\phi)$ of $\phi$ is well defined and $\Lambda(\phi) \neq\{0\}$ implies that the set Fix $(\phi)=\{x \in$ $X: x \in \phi(x)\}$ is non-empty.

Definition 2.4. A metric space $X$ is called a Lefschetz space provided that for each admissible and compact map $\phi: X \multimap \mathrm{X}$ if $\Lambda(\phi)$ is well defined and $\Lambda(\phi) \neq\{0\}$ then $\operatorname{Fix}(\varphi) \neq 0$.

Let $Y$ be a metric space and let $I d_{Y}: Y \rightarrow Y$ be a map given by formula $I d_{Y}(y)=y$ for each $y \in Y$.

Definition 2.5. (see, [4]) A map $r: X \rightarrow Y$ of a space $X$ onto a space $Y$ is said to be an mr-map if there is an admissible map $\phi: Y \rightarrow X$ such that $r \circ \phi=I d_{Y}$. 
Definition 2.6. (see, $[4,6])$ A metric space $X$ is called an absolute multi-retract (notation: $X \in A M R$ ) provided there exists a locally convex space $E$ and an mr-map $r: E \rightarrow$ $X$ from $E$ onto $X$.

Definition 2.7. (see, $[4,6])$ A metric space $X$ is called an absolute neighborhood multi-retract (notation: $X \in A N M R$ ) provided there exists an open subset $U$ of some locally convex space $E$ and an mr-map $r: U \rightarrow X$ from $U$ onto $X$.

Proposition 2.8. (see, $[4,6])$ A space $X$ is an ANMR if and only if there exists a metric space $Z$ and a Vietoris map $p: Z \rightarrow X$ which factors through an open subset $U$ of some locally convex E, i.e., there are two continuous maps $\alpha$ and $\beta$ such that the following diagram is commutative.

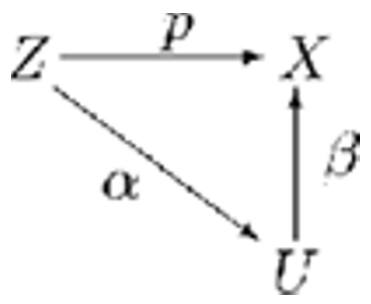

Proposition 2.9. (see, [4]) Let $X \in A N M R$ and let $V \subset X$ be an open set. Then $V \in$ ANMR.

Proposition 2.10. (see, [4]) Assume that $X$ is ANMR. Let $U$ be an open subset in $X$ and $\phi: U \rightarrow U$ be an admissible and compact map, then $\phi$ is a Lefschetz map.

Let $\phi_{X}: X \rightarrow X$ be a map.

$$
\varphi_{X}^{n}= \begin{cases}I d_{X}, & \text { for } n=0, \\ \varphi_{X}, & \text { for } n=1, \\ \varphi_{X} \circ \varphi_{X} \circ \ldots \circ \varphi_{X}(n-\text { iterates }), & \text { for } n>1 .\end{cases}
$$

Definition 2.11. Let $X \subset Q$ be a closed subset, where $Q$ is a Hilbert cube. We say that $X$ is movable in $Q$ provided every neighborhood $U$ of $X$ admits a neighborhood $U^{\prime}$ of $X, U^{\prime} \subset U$, such that for every neighborhood $U^{\prime \prime}$ of $X, U^{\prime \prime} \subset U$, there exists a homotopy $H: U^{\prime} \mathrm{x}[0,1] \rightarrow U$ with $H(x, 0)=x$ and $H(x, 1) \in U^{\prime \prime}$, for any $x \in U^{\prime}$.

Lemma 2.12. (see, [7]) Let $X \subset Q$ be a compact absolute approximative neighborhood retract in the sense of Clapp (in particular $X \in A N R$ or $X \in A R$ ). Then $X$ is movable in $Q$.

The notion of shape is understood in the sense of Borsuk (see, [3]). It can be proven that a compact set of trivial shape is acyclic.

Definition 2.13. (see, [2]) Let $X$ be a continuum space and let $A \subset X$. Then an u.s.c multi-valued map $\sigma: X \rightarrow A$ with compact images is said to be a multi-retraction of $X$ to $A$ if the following conditions are satisfied:

2.13.1 for each $x \in X \sigma(x)$ is a set of a trivial shape,

2.13.2 for each $x \in A x \in \sigma(x)$.

We say that a subset $A$ of a space $X$ is a neighborhood multi-retract in the space $X$ if there is a neighborhood $U$ of $A$ in $X$ and a multi-retraction $\sigma: U \rightarrow A$. If $U=X$, then we call $A$ a multi-retract of the space $X$.

Definition 2.14. (see, [2]) A continuum $X$ will be called an absolute neighborhood multi-retract $(X \in m$ - ANR) provided that, for every continuum $Y \supset X, X$ is a 
neighborhood multi-retract of $Y$. If $X$ is a multi-retract of every space $Y \supset X$, then we call $X$ an absolute multi-retract $(Y \in m-A R)$.

\section{Main result}

First, the notion of the domination of space will be introduced, which is the generalization in the sense of Borsuk as well as in the sense of Suszycki and in the multi-valued sense.

Definition 3.1. We shall say that a metric space $Y$ multi-dominates a metric space $X$ (we write $Y \circ \geq \circ X)$ if there exists multi-valued admissible maps $\phi: Y \rightarrow X$ and $\psi: X \rightarrow Y$ such that the following conditions are satisfied:

3.1.1 for each $x \in X x \in \phi(\psi(x))$,

3.1.2 there exist selected pairs $\left(p_{1}, q_{1}\right) \subset \phi$ and $\left(p_{2}, q_{2}\right) \subset \psi$ such that

$$
I d_{H_{*}(X)}=q_{1_{*}} p_{1_{*}}^{-1} \circ q_{2_{*}} p_{2_{*}}^{-1} .
$$

Let us assume that if $(Y \circ \geq \circ X)$ and if it is not true that $(\mathrm{X} \circ \geq \circ Y)$ then we write $(Y \circ>\circ X)$. The defined domination can be treated as a relation in the class of metric spaces. We will prove it is a transitive relation.

Theorem 3.2. Let $X, Y, Z$ be metric spaces.

$$
\text { If }(Y \circ \geq \circ Z) \text { and }(Z \circ \geq \circ X) \text { then }(Y \circ \geq \circ X) \text {. }
$$

Proof. We have the following diagram:

$$
X \stackrel{\psi_{2}}{\rightarrow} Z \stackrel{\psi_{1}}{\rightarrow} Y \stackrel{\varphi_{1}}{\rightarrow} Z \stackrel{\varphi_{2}}{\rightarrow} X, \text { where }
$$

for each $z \in Z$ and $x \in X z \in \varphi_{1}\left(\psi_{1}(z)\right)$ and $x \in \varphi_{2}\left(\psi_{2}(x)\right)$,

there exist $\left(p_{1}, q_{1}\right) \subset \phi_{1},\left(p_{2}, q_{2}\right) \subset \phi_{2},\left(r_{1}, s_{1}\right) \subset \psi_{1},\left(r_{2}, s_{2}\right) \subset \psi_{2}$ such that

$$
\begin{aligned}
& I d_{H_{*}(Z)}=q_{1_{*}} p_{1_{*}}^{-1} \circ s_{1_{*}} r_{1_{*}}^{-1}, \\
& I d_{H_{*}(X)}=q_{2_{*}} p_{2_{*}}^{-1} \circ s_{2_{*}} r_{2_{*}}^{-1} .
\end{aligned}
$$

We define multi-valued admissible maps $\phi: Y \rightarrow X, \psi: X \multimap Y$ given by formula:

$$
\varphi(\gamma)=\varphi_{2}\left(\varphi_{1}(y)\right) \text { for any } y \in Y, \quad \psi(x)=\psi_{1}\left(\psi_{2}(x)\right) \text { for any } x \in X .
$$

We show that for each $x \in X x \in \phi(\psi(x))$. Let $x \in X$ and let $z \in \psi_{2}(x)$. From (1) we get that $z \in \phi_{1}\left(\psi_{1}(z)\right)$. Hence we have

$$
\psi_{2}(x) \subset \varphi_{1}\left(\psi_{1}\left(\psi_{2}(x)\right)\right) .
$$

It is clear that

$$
x \in \varphi_{2}\left(\psi_{2}(x)\right) \subset \varphi_{2}\left(\varphi_{1}\left(\psi_{1}\left(\psi_{2}(x)\right)\right)\right)=\varphi(\psi(x)) .
$$

From Proposition 2.2 we get that there exist $(p, q) \subset \phi,(r, s) \subset \psi$ such that:

$$
q_{*} p_{*}^{-1}=q_{2_{*}} p_{2_{*}}^{-1} \circ q_{1_{*}} p_{1_{*}}^{-1}, s_{*} r_{*}^{-1}=s_{1_{*}} r_{1_{*}}^{-1} \circ s_{2_{*}} r_{2_{*}}^{-1} .
$$

We have

$$
\begin{gathered}
q_{*} p_{*}^{-1} \circ s_{*} r_{*}^{-1}=\left(q_{2_{*}} p_{2_{*}}^{-1} \circ q_{1_{*}} p_{1_{*}}^{-1}\right) \circ\left(s_{1_{*}} r_{1_{*}}^{-1} \circ s_{2_{*}} r_{2_{*}}^{-1}\right)= \\
=q_{2_{*}} p_{2_{*}}^{-1} \circ\left(q_{1_{*}} p_{1_{*}}^{-1} \circ s_{1_{*}} r_{1_{*}}^{-1}\right) \circ s_{2_{*}} r_{2_{*}}^{-1}=q_{2_{*}} p_{2_{*}}^{-1} \circ s_{2_{*}} r_{2_{*}}^{-1}=I d_{H_{*}(X) .}
\end{gathered}
$$


Let us remind that if a multi-valued mapping u.s.c. $\phi: X \multimap Y$ has compact and acyclic images then:

the set $\left\{q_{*} p_{*}^{-1}:(p, q) \subset \varphi\right\}$ consists of one element (see, [5, p. 163]).

Another fact that shall be proven is the following:

Proposition 3.3. Let $X$ be a continuum and acyclic space and let $Y$ be a metric space.

Then $Y \circ \geq \circ X$.

Proof. Let $y_{0} \in Y$ be a stationary point. We define maps $\phi: Y \rightarrow X$ given by $\phi(y)=X$ for each $y \in Y$ and $\psi: X \rightarrow Y$ given by $\psi(x)=y_{0}$ for each $x \in X$. Obviously for any $x \in X$

$$
x \in X=\varphi(\psi(x)) .
$$

The images of a map $\phi \circ \psi$ are compact and acyclic. Hence and from Proposition 2.2 and (4) we get the condition 3.1.2.

Let us notice that from 3.1.2 it follows that

$$
q_{2 *} p_{2_{*}}^{-1}: H_{*}(X) \rightarrow H_{*}(Y) \text { is a monomorphism. }
$$

Hence and from 3.3 we have:

Proposition 3.4. Let $X$ be a continuum and acyclic space and let $Y$ be a metric and non-acyclic space. Then $Y \circ>\circ X$.

Particularly from the last fact it follows that

$$
\mathbb{S}^{n-1} \circ>\circ \mathbb{K}^{n},
$$

where $\mathbb{S}^{n-1}$ is a $(n-1)$-dimensional sphere and $\mathbb{K}^{n}$ is an $n$-dimensional ball in a Euclidean space $\mathbb{R}^{n}$, for every $n \in N$.

Example 3.5. Let $Y \subset Q$ be an acyclic continuum and such that $Y$ is not movable (see, [8]), where $Q$ is a Hilbert cube. From Proposition 3.3 we get $Q \circ \geq \circ Y$. We show that it is not true that $Q \geq Y$. If the space $Q$ dominated the space $Y$ in the sense of Borsuk, then $Y \in A R$, however, every absolute retract is movable (see, Lemma 2.12) and it would contradict the fact that $Y$ is not movable.

Let us introduce some notation. $Y \circ \geq X$ will mean that the mapping $\psi=g: X \rightarrow Y$ can be chosen single-valued (see, Definition 3.1), whereas $Y \geq 0 X$ will mean that the mapping $\phi=r: Y \rightarrow X$ is single-valued. Then $Y \geq \circ_{s} X$ will denote that $\phi=r: Y \rightarrow X$ is single-valued and the conditions 3.1.1 and 3.1.2 will be replaced by a stronger condition:

$$
\text { for each } x \in X r(\psi(x))=\{x\} \text {. }
$$

It can be easily proven that each of these relations, i.e., $0 \geq 2 \geq 0, \geq 0_{s}$ is transitive.

Obviously we have:

$$
\begin{aligned}
& \operatorname{if}(Y \circ \geq X \text { or } Y \geq \circ X) \text { then } Y \circ \geq \circ X, \\
& \text { if } Y \geq \circ_{S} X \text { then } Y \geq \circ X \text {. }
\end{aligned}
$$

Remark 3.6. The relation $Y \circ_{s} \geq X$, i.e., $\psi=g: X \rightarrow Y$ (see, Definition 3.1) is a singlevalued and continuous map and the conditions 3.1.1 and 3.1.2 are replaced by a stronger condition:

$$
\text { for each } x \in X \varphi(g(x))=\{x\},
$$


is not considered separately since we treat the relation $Y \circ \geq X$ as an integral whole according to the earlier articles on the subject (see, [2]) and the Theorem 3.14 (see, 3.14.5 and 3.14.6).

The next two examples show that neither $Y \circ \geq X$ nor $Y \geq \circ X$ retains Lefschetz property.

Example 3.7. Let $Q$ be a Hilbert cube and let $X \subset Q$ be an acyclic continuum and such that it is not a Lefschetz space (see, [9]). We observe, that $Q \circ \geq X$ (see, Proposition 3.3). $Q$ is a Lefschetz space and $X$ is not a Lefschetz space.

Example 3.8. Let $S=\left\{x \in \mathbb{R}^{2} \mid\|x\|=1\right\}$ be the unit circle. Let $\alpha:[0, \infty) \rightarrow \mathbb{R}^{2}$ be a function defined as follows:

$$
\alpha(t)=\left(\left(1+e^{-t}\right) \cos (t),\left(1+e^{-1}\right) \sin (t)\right)
$$

for any $t \geq 0$. Let $X:=\alpha([2, \infty))$. Let $Y:=S \cup X$ and $Z=((S \cup([2, \infty) \times\{0\})) \times I)$.

We can regard $Y$ as a subset of $\mathbb{R}^{2} \times\{0\} \subset \mathbb{R}^{3}$. Let $C Y:=Y \times I / \sim$ be the cone over $Y$ and vertex $v=(0,0,1)$. It is not hard to show that $C Y$ is contractible to the point $v$. Moreover, Knill showed (see, [9]) that CY is not a fixed point space! Define a function

$$
p: Z \rightarrow C Y
$$

by $p(z, s)=[\beta(z), s]$, where $\beta: Z \rightarrow Y$ is given by the following formula

$$
\beta(z)=\left\{\begin{array}{cl}
z & \text { if } z \in S \\
\alpha(t) & \text { if } z=(t, 0) \in[2, \infty) \times\{0\} .
\end{array}\right.
$$

It is clear that $p(Z)=C Y$. We define a map $\psi: C Y \rightarrow Z$ given by formula $\psi(y)=Z$ for all $y \in C Y$. The mapping $\psi$ is admissible because the pair $(I d, f) \subset \phi$, where the mapping Id :CY $\rightarrow C Y$ is given by the formula $I d(y)=y$ for every $y \in C Y$ and the mapping $f: C Y \rightarrow Z$ is constant. We show that

$$
Z \geq \circ C Y \text {. }
$$

We observe that for each $y \in C Y y \in p(\psi(y))=C Y$. The images of a map $p \circ \psi$ are compact and acyclic. Hence, and from Proposition 2.2 and (4), we get the condition 3.1.2. The space $Z \in A N R$, so $Z$ is a Lefschetz space and $C Y$ is not a Lefschetz space.

From the above two examples, it follows that the dominations of $0 \geq$ and $\geq 0$ type do not preserve a fixed point property. However, the following fact is true:

Theorem 3.9. Let $Y$ be a Lefschetz space and let $Y \geq 0_{s} X$, where $X$ is a metric space.

Then $X$ is a Lefschetz space.

Proof. Let $\psi: X \rightarrow Y$ be an admissible map, $r: Y \rightarrow X$ be a continuous map and such that

$$
\text { for each } x \in X r(\psi(x))=\{x\} \text {. }
$$

Let $\phi: X \nrightarrow X$ be an admissible and compact map. It is clear that the following diagram:

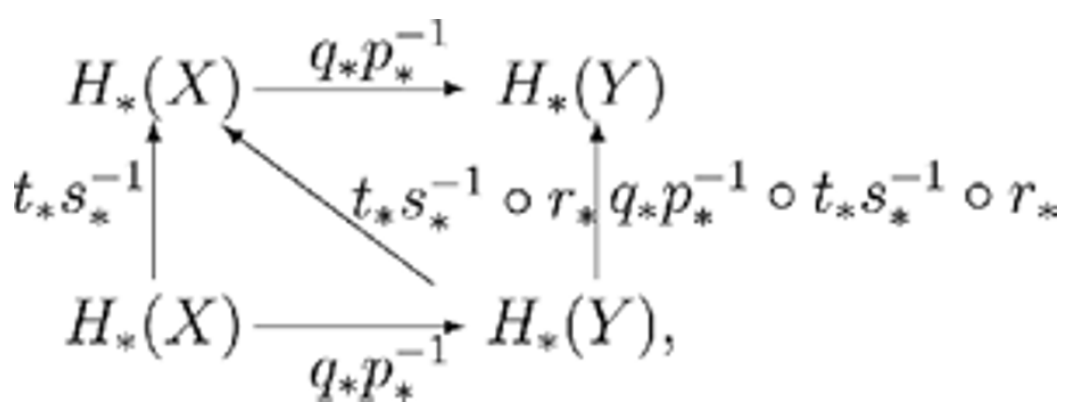


is commutative, where $(p, q) \subset \psi$ and $(s, t) \subset \phi$. From (10) we get that if $y \in\left(q p^{-1} \circ\right.$ $\left.t s^{-1} \circ r\right)(y)$, then $r(y) \in\left(\left(r \circ q p^{-1}\right) \circ t s^{-1}\right)(r(y))=\left(t s^{-1}\right)(r(y)) \subset \phi(r(y))$. Hence $\operatorname{Fix}(\varphi) \neq \emptyset$.

The next example shows that the domination of $\geq 0_{s}$ type is more general than $\geq$ type (in the sense of Borsuk).

Example 3.10. Let $Y \subset Q$ be an acyclic continuum and such that $Y$ is not movable. Let $p: Q \rightarrow Y$ be a Vietoris map (see, [8]). Then $Q \geq 0_{s} Y$, where $r=p: Q \rightarrow Y$ and $\psi$ $: Y \rightarrow Q$ given by formula $\psi(y)=p^{-1}(y)$ for each $y \in Y$. Obviously the space $Q$ does not dominate the space $Y$ in the sense of Borsuk because otherwise $Y$ would have to be an absolute retract, and thus, in particular, a movable space, and that would contradict the fact that $Y$ is not movable.

Let us notice that from Proposition 3.3 and Example 3.8, the relation $\circ \geq 0$ (and even $\geq 0$ ) is more general than the relation $\geq 0_{s}$. Now a theorem will be given that will be the summary of the above considerations. First a more general version of Suszycki retract (see, [2]) shall be given using admissible mappings for its definition.

Definition 3.11. We shall say that a set $A \subset X$ is an admissible multi-retract of $X$ if there exists an admissible map $\phi: X \rightarrow A$ such that the following conditions are satisfied:

3.11.1 for each $x \in A x \in \phi(x)$,

3.11.2 there exist $(p, q) \subset \phi$ such that $I d_{H_{*}(A)}=q_{*} p_{*}^{-1} \circ i_{*}$ where $i: A \rightarrow X$ is an inclusion.

The mapping $\phi$ will be called an admissible multi-retraction.

It is not difficult to notice that if $A \subset X$ is an admissible multi-retract of $X$ then $X \circ$ $\geq A$. Moreover, if $A \subset X$ is a multi-retract $X$ in the sense of Suszycki (see, Definition 2.14), then it is its admissible multi-retract.

Definition 3.12. A space $X$ is an admissible absolute multi-retract (am - AR) if for each metric space $Y$ and homeomorphism $h: X \rightarrow Y$, where $h(X)$ is a closed subset in $Y, h(X)$ is an admissible multi-retract of $Y$.

Definition 3.13. A space $X$ is an admissible absolute neighborhood multi-retract (am - ANR) if for each metric space $Y$ and homeomorphism $h: X \rightarrow Y$, where $h(X)$ is a closed subset in $Y, h(X)$ is an admissible multi-retract of some $U$, where $U \subset Y$ is an open set and $h(X) \subset U$.

Obviously, $A R \subset m-A R \subset$ am $-A R$ and $A N R \subset m-A N R \subset$ am $-A N R$ (see, Definition 2.14).

Theorem 3.14. Let $X$ be a metric space. There exists a locally convex space $E$ and an open set $U \subset E$ such that:

3.14.1 $(E \geq X) \Leftrightarrow(X \in A R)$,

3.14.2 $(U \geq X) \Leftrightarrow(X \in A N R)$,

3.14.3 $\left(E \geq \circ_{s} X\right) \Leftrightarrow(X \in A M R)$,

3.14.4 $\left(U \geq O_{s} X\right) \Leftrightarrow(X \in A N M R)$,

3.14.5 $(E \circ \geq X) \Leftrightarrow(X \in a m-A R)$,

3.14.6 $(U \circ \geq X) \Leftrightarrow(X \in a m-A N R)$.

Proof. The proofs 3.14.1 and 3.14.2 are known from the mathematic literature in the case of $E$ being a normalized space. The situations where $E$ is a locally convex space, but not necessarily normalized, is considered in (see, [6, Theorem 3.6]). Then the proofs 3.14.3 and 3.14.4 follow directly from the $A M R$ and $A N M R$ definitions (see, Definitions 2.6 and 2.7). We will prove the condition 3.14.6, since the condition 3.14.5 
can be proven in a similar way. We assume that there exists a locally convex space and an open set $U \subset E$ such that $U \circ \geq X$. Then there exists a continuous map $g: X \rightarrow U$ and an admissible map $\phi: U \rightarrow \mathrm{X}$ such that

for each $x \in X x \in \varphi(g(x))$,

$$
\text { there exists }(p, q) \subset \varphi I d_{H_{*}(X)}=q_{*} p_{*}^{-1} \circ q_{* \prime}
$$

Let $h: X \rightarrow Y$ be a homeomorphism such that $h(X) \subset Y$ is a closed set. Choose the continuous extension $r: Y \rightarrow E$ of a map $g \circ h^{-1}: h(X) \rightarrow U \subset E$. Let $V=\mathrm{r}^{-1}(U)$. Then $h(X) \subset V$. We define $\psi: V \rightarrow h(X)$ given by the formula $\psi=h \circ \phi \circ r_{V}$, where $r_{V}: V \rightarrow U$ given by $r_{V}(x)=r(x)$ for each $x \in V$. We show that for each $y \in h(X) y \in$ $\psi(y)$. Let $y \in h(X)$. Then $r_{V}(y)=g\left(h^{-1}(y)\right)$ and from $(11) h^{-1}(y) \in \phi\left(g\left(h^{-1}(y)\right)\right)$. Hence

$$
y \in h\left(\varphi\left(g\left(h^{-1}(\gamma)\right)\right)\right)=h\left(\varphi\left(r_{V}(\gamma)\right)\right)=\psi(y) .
$$

From (12) we have

$$
h_{*} \circ q_{*} p_{*}^{-1} \circ\left(r_{V}\right)_{*} \circ i_{*}=h_{*} \circ q_{*} p_{*}^{-1} \circ\left(g \circ h^{-1}\right)_{*}=h_{*} \circ\left(q_{*} p_{*}^{-1} \circ g_{*}\right) \circ h_{*}^{-1}=I d_{H_{*}(h(x))^{\prime}}
$$

where $i: h(X) \rightarrow V$ is an inclusion.

Assume now that $X \in a m-A N R$. Let $\psi: U \rightarrow h(X)$ be an admissible multi-retraction, where $U \subset Y$ is an open set, $h: X \rightarrow Y$ homeomorphism, $h(X)$ is a closed set in $Y$ and $h(X) \subset U$. Then we have

$$
\begin{aligned}
& \text { for each } y \in h(X) y \in \psi(y), \\
& \text { there exists }(p, q) \subset \psi I d_{H_{*}(h(X))}=q_{*} p_{*}^{-1} \circ j_{* \prime}
\end{aligned}
$$

where $j: h(X) \rightarrow U$ is an inclusion. We define a continuous map $g: X \rightarrow U$ given by formula $g(x)=j(h(x))$ for each $x \in X$ and an admissible map $\phi: U \rightarrow X$ given by $\phi(x)$ $=h^{-1}(\psi(x))$ for each $x \in U$. Obviously $x \in \phi(g(x))$ for each $x \in X$. From (14) we get

$$
h_{*}^{-1} \circ\left(q_{*} p_{*}^{-1} \circ j_{*}\right) \circ h_{*}=I d_{H_{*}(X)} .
$$

Remark 3.15. In Theorem 3.14 (3.14.5 and 3.14.6), a locally convex space can be replaced by a normed space, without loss of generality (see, [6, Theorem 3.6]).

Multi-valued domination in the sense of Suszycki was determined in the class of compact metric spaces. Multi-domination defined in this article (see, Definition 3.1) encompasses a broader class of metric spaces, not necessarily compact, which is illustrated by the following example:

Example 3.16. Let $X$ be a non-compact metric space and such that $X \in A N M R$ and $X \notin A N R$ (see, [4]). Then there exists a normed space $E$, an open set $U \subset E$, an admissible map $\psi: X \rightarrow U$ and a continuous map $r: U \rightarrow X$ such that $r(\psi(x))=\{x\}$ for each $x$ $\in X\left(U \geq 0_{s} X\right)$. Let $Y$ be a compact metric space and such that $Y \in m-A N R$ and $Y \notin$ ANR (see, [2]) $(V \circ \geq Y)$ (see, Theorem 3.14). Then there exists an open set $V \subset Q$, a continuous map $g: Y \rightarrow V$, a multi-valued map $\phi: V \rightarrow Y$ such that $y \in \phi(g(y))$ for each $y \in Y$, where $\phi(x)$ is a compact and of trivial shape for any $x \in V$. Obviously $(X \times$ $Y) \notin m$ - ANR, since the space $X \times Y$ is non-compact from assumption. We show that

$$
(U \times V) \circ \geq \circ(X \times Y) .
$$


We have

$$
X \times Y \stackrel{\psi \times g}{\longrightarrow} U \times V \stackrel{r \times \varphi}{\longrightarrow} X \times Y .
$$

Hence

$$
(r \times \varphi)(\psi \times g)(x, y)=x \times \varphi(g(y)), \text { for each }(x, y) \in(X \times Y) .
$$

For each $(x, y) \in(X \times Y)$ the set $x \times \phi(g(y))$ is a compact and of trivial shape and ( $x$, $y) \in(x \times \phi(g(y)))$. Hence, and from (4), the conditions of the Definition 3.1 are satisfied.

The last example also shows that the Definition 3.1 is necessary because we certainly know that $(U \times V) \circ \geq \circ(X \times Y)$. However, the following open problem still remains:

Open problem 3.17. Are there metric spaces $X, Y$ such that $Y \circ \geq 0 X$, but it is false that $Y \circ \geq \circ X$.

\section{Authors' contributions}

No authors contributions. I am the solely author of it, I performed all the necessary studies and wrote the entire article.

\section{Competing interests}

The authors declare that they have no competing interests.

Received: 13 August 2011 Accepted: 22 February 2012 Published: 22 February 2012

\section{References}

1. Borsuk, K: Theory of Retracts, Monografie Matematyczne. Tom 44, PWN, Warsaw (1967)

2. Suszycki, A: Retracts and homotopies for multi-maps. Fundam Math. 115(1), 9-26 (1983)

3. Borsuk, K: Theory of Shape, Monografie Matematyczne. Tom 59, PWN, Warsaw (1975)

4. Skiba, R, Ślosarski, M: On a generalization of absolute neighborhood retracts. Topol Appl. 156, 697-709 (2009). doi:10.1016/j.topol.2008.09.007

5. Górniewicz, L: Topological Fixed Point Theory of Multivalued Mappings. Springer (2006)

6. Ślosarski, M: On a generalization of approximative absolute neighborhood retracts. Fixed Point Theory. 10(2), 329-346 (2009)

7. Bogatyi, SA: Approximative and fundamental retracts. Math USSR Sb. 22, 91-103 (1974). doi:10.1070/ SM1974v022n01ABEH001687

8. Keesling, JE: A non-movable trivial shape decomposition of the Hilbert Cube. Bull Acad Polon Sci Sér Sci Math Astronom Phys. 23, 997-998 (1975)

9. Knill, RJ: Cones, products and fixed points. Fund Math. 60, 35-46 (1967)

doi:10.1186/1687-1812-2012-23

Cite this article as: Ślosarski: The multi-valued domination of metric spaces. Fixed Point Theory and Applications 2012 2012:23.

\section{Submit your manuscript to a SpringerOpen ${ }^{\circ}$} journal and benefit from:

- Convenient online submission

Rigorous peer review

- Immediate publication on acceptance

- Open access: articles freely available online

- High visibility within the field

- Retaining the copyright to your article

Submit your next manuscript at $>$ springeropen.com 